\title{
Recognition of 27-Class Protein Folds by Adding the Interaction of Segments and Motif Information
}

\author{
Zhenxing Feng and Xiuzhen Hu \\ Department of Sciences, Inner Mongolia University of Technology, Hohhot, China \\ Correspondence should be addressed to Xiuzhen Hu; hxz@imut.edu.cn
}

Received 27 November 2013; Accepted 28 June 2014; Published 21 July 2014

Academic Editor: Huseyin Seker

Copyright ( 2014 Z. Feng and X. Hu. This is an open access article distributed under the Creative Commons Attribution License, which permits unrestricted use, distribution, and reproduction in any medium, provided the original work is properly cited.

\begin{abstract}
The recognition of protein folds is an important step for the prediction of protein structure and function. After the recognition of 27class protein folds in 2001 by Ding and Dubchak, prediction algorithms, prediction parameters, and new datasets for the prediction of protein folds have been improved. However, the influences of interactions from predicted secondary structure segments and motif information on protein folding have not been considered. Therefore, the recognition of 27-class protein folds with the interaction of segments and motif information is very important. Based on the 27-class folds dataset built by Liu et al., amino acid composition, the interactions of secondary structure segments, motif frequency, and predicted secondary structure information were extracted. Using the Random Forest algorithm and the ensemble classification strategy, 27-class protein folds and corresponding structural classification were identified by independent test. The overall accuracy of the testing set and structural classification measured up to $78.38 \%$ and $92.55 \%$, respectively. When the training set and testing set were combined, the overall accuracy by 5 -fold cross validation was $81.16 \%$. In order to compare with the results of previous researchers, the method above was tested on Ding and Dubchak's dataset which has been widely used by many previous researchers, and an improved overall accuracy $70.24 \%$ was obtained.
\end{abstract}

\section{Introduction}

With the accomplishment of the Human Genome Project, the "post genome era" has presented a large number of protein sequences that have challenged to develop a high-throughput computational method to structurally annotate the sequences coming from genomic data. One of these critical structures, the protein fold, reflects a key topological structure in proteins as it contains three major aspects of protein structure: the units of secondary structure, the relative arrangement of structures, and the overall relationship of protein peptide chains [1].

A protein can only perform its physiological functions if it folds into its proper structure. Abnormal protein folding causes molecular aggregation, precipitation, or abnormal transport, resulting in different diseases. For example, the prion protein (PRNP) accumulates in the brain and causes neurodegenerative diseases, such as scrapie, CreutzfeldtJakob disease, Parkinson's disease, Huntington's disease, and mad cow disease. And the PRNP is a pathogenic protein caused by the abnormal folding of proteins. Thus, the correct identification of protein folds can be valuable for the studies on pathogenic mechanisms and drug design [2-8]. Thus, the identification of folding types is a highly important research project in bioinformatics.

Proteins with similar sequences tend to fold into similar spatial structures. When proteins have close evolutionary relationships, the similarity-based methods can achieve very reliable predicted results $[9,10]$. However, they are unreliable for the identification of proteins with far evolutionary relationships. Fortunately, approaches that extract relevant features from protein sequences to identify protein folds have made significant advance in recent years. In 2001, Ding and Dubchak [11] built the 27-class folds dataset and used multiple feature groups as parameters, including amino acid composition, predicted secondary structure, hydrophobicity, Van der Waals volume, polarity, and polarizability; then they proposed support vector machines and neural networks methods for the identification of 27-class protein folds. The best overall accuracy achieved was $56 \%$. 
Afterwards, based on the dataset built by Ding and Dubchak and the feature parameters, some researchers suggested algorithm improvements for the identification of folds. For example, Chinnasamy et al. [12] introduced the phylogenetic tree and Bayes classifier for the identification of protein folds and structural classifications and achieved an overall accuracy of $58.18 \%$ and $80.52 \%$, respectively. Nanni [13] proposed a new ensemble of K-local hyperplane based on random subspace and feature selection and achieved an overall accuracy of $61.1 \%$. Guo and Gao [14] presented a novel hierarchical ensemble classifier named GAOEC (GeneticAlgorithm Optimized Ensemble Classifier) and achieved an overall accuracy of $64.7 \%$. Damoulas and Girolami [15] offered the kernel combination methodology for the prediction of protein folds and the best accuracy was 70\%. Lin et al. [16] exploited novel techniques to impressively increase the accuracy of protein fold classification.

Based on the dataset built by Ding and Dubchak, other previous researchers suggested the selection of feature parameters for the identification of protein folds. For example, Shamim et al. [17] used the feature parameters of structural properties of amino acid residues and amino acid residue pairs and achieved an overall accuracy of $65.2 \%$. Dong et al. [18] proposed a method called ACCFold and achieved an overall accuracy of $70.1 \%$. Li et al. [19] proposed a method called PFP-RFSM and got improved results for the identification of protein folds.

Based on the dataset built by Ding and Dubchak [11], many pioneering researchers have not only focused on the selection of feature parameters but also on the improvement of algorithms to identify protein folds. For example, Zhang et al. [20] in our group proposed an approach of increment of diversity by selecting the pseudo amino acid composition, position weight matrix score, predicted secondary structure, and the second neighbor dipeptide composition; by using the characteristic parameters to predict 27-class protein folds and structural classifications, the overall accuracies measured up to $61.10 \%$ and $79.11 \%$, respectively. Shen and Chou [21] applied the OET-KNN ensemble classifier to identify folding types by introducing pseudo amino acid with sequential order information as a feature parameter and achieved an overall accuracy of $62.1 \%$. Chen and Kurgan [22] proposed the PFRES method using evolutionary information and predicted secondary structure, obtaining an accuracy of $68.4 \%$. Ghanty and Pal [23] proposed the Fusion of Heterogeneous Classifiers approach, with features including the selected trio AACs and trio potential, and the overall recognition accuracy reached 68.6\%. Shen and Chou [24] applied a method to identify protein folds by using functional domain and sequential evolution information and achieved an overall accuracy of 70.5\%. Yang et al. [25] proposed a novel ensemble classifier called MarFold, which combines three margin-based classifiers for protein folds recognition, and the overall prediction accuracy was $71.7 \%$.

Other previous researchers have constructed new 27class fold datasets and performed corresponding researches. For example, based on the Astral SCOP 1.71, with sequence identity below $40 \%$, Shamim et al. [17] constructed a dataset including 2554 proteins belonging to 27-class folds, proposed structural properties of amino acid residues and amino acid residue pairs as parameters, and achieved an overall accuracy of $70.5 \%$ by 5 -fold cross validation. Based on the Astral SCOP 1.73 with sequence identity below $40 \%$, Dong et al. [18] constructed the 27-class folds dataset which contains 3202 sequences, proposed an ACCFold method, and obtained an overall accuracy of $87.6 \%$ by 5 -fold cross validation. According to Ding and Dubchak's [11] description on the construction of protein folds dataset in literature, based on the Astral SCOP 1.75, Liu and $\mathrm{Hu}$ [26] in our group constructed an expanded 27-class folds dataset. The dataset with a sequence identity below 35\% contains 1895 sequences. Motif frequency, low-frequency power spectral density, amino acid composition, predicted secondary structure, and autocorrelation function values were combined as feature parameters to identify the 27-class protein folds and structural classifications, and the overall accuracy by independent test was $66.67 \%$ and $89.24 \%$, respectively. At the same time, researches on 76 -class folds, 86-class folds, and 199-class folds have also made some progress $[18,27]$.

In this paper, based on the dataset built by Liu et al. [27] in our group, amino acid composition, motif frequency, predicted secondary structure information, and the interaction of predicted secondary structure segments were applied for the recognition of protein folds. These features reflect the sequence information, structural information, and functional information. Based on the ensemble classification strategy with the Random Forest algorithm, improved identification results of 27-class protein folds and structural classifications were achieved.

\section{Materials and Methods}

2.1. Protein Folds Dataset. The dataset used in this paper was built by Liu et al. [27] in our group. The sequence identity of the dataset was below 35\%. The number of sequences in each fold was not less than 10 . The training set and testing set contained 956 protein chains and 939 protein chains, respectively. The distribution of corresponding folds name, number of sequences, and structural class is shown in Table 1 . The other dataset used in this paper was built by Ding and Dubchak and reorganized by Shen and Chou [21]. The dataset with sequence identity below $35 \%$ used by many researchers contained a training set including 311 protein chains and a testing set including 383 protein chains. The distribution information of corresponding protein folds is also shown in Table 1. The dataset is available at the following website: http://202.207.29.245:8080/Ha/HomePage/fzxHomePage.jsp.

The dataset built in our group was according to Ding and Dubchak's description about the construction of protein folds dataset in literature [11]. The number of sequences in the dataset was three times greater than Ding and Dubchak's dataset [11].

\subsection{The Selection of Feature Parameters}

2.2.1. Amino Acid Composition (A). The distributions of the 20 amino acid residues in protein sequences for different protein folds are obviously different, and previous researches 
TABLE 1: Datasets of 27-class protein folds.

\begin{tabular}{|c|c|c|c|c|}
\hline \multirow[t]{2}{*}{ Fold } & \multicolumn{2}{|c|}{ Dataset built by Liu et al. [27] } & \multicolumn{2}{|c|}{$\begin{array}{c}\text { Dataset built by Ding and } \\
\text { Dubchak [11] }\end{array}$} \\
\hline & Training set & Testing set & Training set & Testing set \\
\hline All $\alpha$ structural class & 174 & 169 & 54 & 61 \\
\hline (1) Globin-like & 14 & 14 & 13 & 6 \\
\hline (2) Cytochrome c & 10 & 10 & 7 & 9 \\
\hline (3) DNA-binding 3-helical bundle & 92 & 90 & 12 & 20 \\
\hline (4) 4-helical up-and-down bundle & 25 & 24 & 7 & 8 \\
\hline (5) 4-helical cytokines & 8 & 8 & 9 & 9 \\
\hline (6) Alpha; EF-hand & 25 & 23 & 6 & 9 \\
\hline All $\beta$ structural classes & 260 & 254 & 109 & 117 \\
\hline (7) Immunoglobulin-like $\beta$-sandwich & 86 & 85 & 30 & 44 \\
\hline (8) Cupredoxins & 18 & 18 & 9 & 12 \\
\hline (9) Viral coat and capsid proteins & 24 & 24 & 16 & 13 \\
\hline (10) ConA-like lectins/glucanases & 18 & 17 & 7 & 6 \\
\hline (11) SH3-like barrel & 41 & 41 & 8 & 8 \\
\hline (12) OB-fold & 29 & 28 & 13 & 19 \\
\hline (13) Trefoil & 11 & 10 & 8 & 4 \\
\hline (14) Trypsin-like serine proteases & 17 & 16 & 9 & 4 \\
\hline (15) Lipocalins & 16 & 15 & 9 & 7 \\
\hline$\alpha / \beta$ structural class & 341 & 337 & 115 & 143 \\
\hline (16) (TIM)-barrel & 93 & 92 & 29 & 48 \\
\hline (17) FAD (also NAD)-binding motif & 5 & 5 & 11 & 12 \\
\hline (18) Flavodoxin-like & 37 & 36 & 11 & 13 \\
\hline (19) NAD(P)-binding Rossmann-fold & 17 & 16 & 13 & 27 \\
\hline (20) P-loop-containing nucleotide & 74 & 73 & 10 & 12 \\
\hline (21) Thioredoxin-like & 37 & 36 & 9 & 8 \\
\hline (22) Ribonuclease H-like motif & 39 & 40 & 10 & 12 \\
\hline (23) Hydrolases & 33 & 33 & 11 & 7 \\
\hline (24) Periplasmic binding protein-like & 6 & 6 & 11 & 4 \\
\hline$\alpha+\beta$ structural class & 181 & 179 & 33 & 62 \\
\hline (25) $\beta$-Grasp & 39 & 39 & 7 & 8 \\
\hline (26) Ferredoxin-like & 101 & 99 & 13 & 27 \\
\hline (27) Small inhibitors, toxins, and lectins & 41 & 41 & 13 & 27 \\
\hline Overall & 956 & 939 & 311 & 383 \\
\hline
\end{tabular}

have shown that amino acid composition is associated with protein folding information [11, 15, 17, 22]. In this paper, we extracted the occurrence frequencies of 20 amino acid residues in protein sequences; thus we got a 20 -dimensional vector. Thus amino acid composition was proposed as a feature parameter $(\mathrm{A})$.

2.2.2. Motif Information (M). A motif is the conserved local region in a protein during evolution [28], which often has a relationship with biological functions. For example, some motifs are related to DNA-binding sites and enzyme catalytic sites [29]. As feature parameters, motif information has been successfully applied for the prediction of super family, protein folds, and so forth $[27,28,30]$. Two kinds of motifs were used in this paper: one with biological functions obtained by searching the existing functional motif dataset PROSITE [31] and the other with statistically significant motifs searched by MEME (http://meme.nbcr.net/meme/cgi-bin/meme.cgi). 
Motif information (M) includes functional motifs and statistical motif.

(1) Functional Motif. The PROSITE dataset was used to gather protein sequence patterns with notable biological functions. PS_SCAN packets provided by the PROSITE dataset and compiled by a Perl program were used as a motif-scan tool to search the sequences of 27-class folds training set; 45 functional motifs were obtained and selected. For an arbitrary sequence in dataset, the frequencies of different motifs in the sequence were recorded. If a motif occurred once, the corresponding frequency value was recorded as "1"; if the motif occurred twice, the value would be 2 , and so on; otherwise if the motif did not occur, the corresponding frequency value was recorded as " 0 ." Thus, the frequencies of different functional motifs in a protein sequence were converted into a 45-dimensional vector.

(2) Statistical Motif. For statistical motifs, MEME was applied as the motif-scan tool. MEME has been widely used to search protein motifs and DNA sequences [32]. In this paper, the motifs with the three highest frequencies in each kind of folds were selected. Each motif contains 6-10 amino acid residues; thus 81 motifs were obtained from the 27-class folds training set. For an arbitrary sequence in dataset, when a motif occurred once, the frequency value was recorded as "1"; if the motif occurred twice, the value would be 2 , and so on; otherwise if the motif did not occur, the corresponding frequency value was recorded as " 0 ." Thus, frequencies of different statistical motifs in a protein sequence were converted into an 81-dimensional vector.

2.2.3. The Interaction of Segments (ACC). A previous study showed that predicted secondary structure information is a main feature parameter for the identification of multiclass protein folds $[11,17,21,22]$. Here, the online web server PSIPRED (http://bioinf.cs.ucl.ac.uk/psipred/) was used as the tool to obtain the predicted secondary structure of each protein sequence in the 27-class protein fold dataset. As a protein fold is a description based on the secondary structure, the interaction between secondary structures plays an important part on the folding of protein. In this paper, we extracted the average interactions between predicted secondary structure segments as a feature parameter for the recognition of protein folds.

(1) The Calculation of Autocross Covariance (ACC). The autocross covariance (ACC) [33] has been successfully adopted by many researchers for the prediction of protein folds [18], G-proteins [34, 35], protein interaction predictions [36], and $\beta$-hairpins [37]. However, ACC has mainly been used on the research between residues or bases. In this paper, the ACC was used at the level of predicted secondary structure segments (helix, strand, or coil) for the first time to predict protein folds. ACC contains two kinds of variables: the AC variable measures the correlation of the same property (the same property means that two secondary structure segments are the same type) and the CC variable measures the
TABLE 2: The physicochemical property values for 20 amino acid residues.

\begin{tabular}{lcccc}
\hline Code & $\mathrm{H}_{1}$ & $\mathrm{H}_{2}$ & $\mathrm{PL}$ & SASA \\
\hline $\mathrm{A}$ & 0.62 & -0.5 & 8.1 & 1.181 \\
$\mathrm{C}$ & 0.29 & -1 & 5.5 & 1.461 \\
$\mathrm{D}$ & -0.9 & 3 & 13 & 1.587 \\
$\mathrm{E}$ & -0.74 & 3 & 12.3 & 1.862 \\
$\mathrm{~F}$ & 1.19 & -2.5 & 5.2 & 2.228 \\
$\mathrm{G}$ & 0.48 & 0 & 9 & 0.881 \\
$\mathrm{H}$ & -0.4 & -0.5 & 10.4 & 2.025 \\
$\mathrm{I}$ & 1.38 & -1.8 & 5.2 & 1.81 \\
$\mathrm{~K}$ & -1.5 & 3 & 11.3 & 2.258 \\
$\mathrm{~L}$ & 1.06 & -1.8 & 4.9 & 1.931 \\
$\mathrm{M}$ & 0.64 & -1.3 & 5.7 & 2.034 \\
$\mathrm{~N}$ & -0.78 & 2 & 11.6 & 1.655 \\
$\mathrm{P}$ & 0.12 & 0 & 8 & 1.468 \\
$\mathrm{Q}$ & -0.85 & 0.2 & 10.5 & 1.932 \\
$\mathrm{R}$ & -2.53 & 3 & 10.5 & 2.56 \\
$\mathrm{~S}$ & -0.18 & 0.3 & 9.2 & 1.298 \\
$\mathrm{~T}$ & -0.05 & -0.4 & 8.6 & 1.525 \\
$\mathrm{~V}$ & 1.08 & -1.5 & 5.9 & 1.645 \\
$\mathrm{~W}$ & 0.81 & -3.4 & 5.4 & 2.663 \\
$\mathrm{Y}$ & 0.26 & -2.3 & 6.2 & 2.368 \\
\hline
\end{tabular}

correlation of the different properties. In this paper, given the corresponding predicted secondary structure segments (helix, strand, or coil) in one sequence, AC variables describe the average interactions in the same type of predicted secondary structure segments, and the separation distance between two predicted secondary structure segmentsis $\mathrm{lg}$ segments. For example, if two segments are neighboring, then $\lg =1$; if the two segments are next-to-neighboring, then $\lg =2$, and so on. The AC variables are calculated according to the following equation:

$$
\operatorname{AC}(i, \lg )=\sum_{j=1}^{L-\lg } \frac{\left(S_{i, j}-\bar{S}_{i}\right)\left(S_{i, j+\lg }-\bar{S}_{i}\right)}{(L-\lg )} \quad(i=1,2,3),
$$

where $\bar{S}_{i}=\sum_{j=1}^{L} s_{i, j} / L, i$ is one kind of secondary structure segment (helix, strand, or coil), $L$ is the number of the secondary structure segments in a protein sequence, and $S_{i, j}$ is a property value of secondary structure segment $i$ at position $j$. $\bar{S}_{i}$ is the average property value for segment $i$ along the whole sequence.

For example, given the hydrophobicity values (Table 2) for 20 amino acid residues, the secondary structure segment $i$ contains $m$ residues, and $S_{i, j}$ represents the summation of hydrophobic values for the $m$ residues. The dimension of AC variables is $3 * \max (\mathrm{lg})$. 


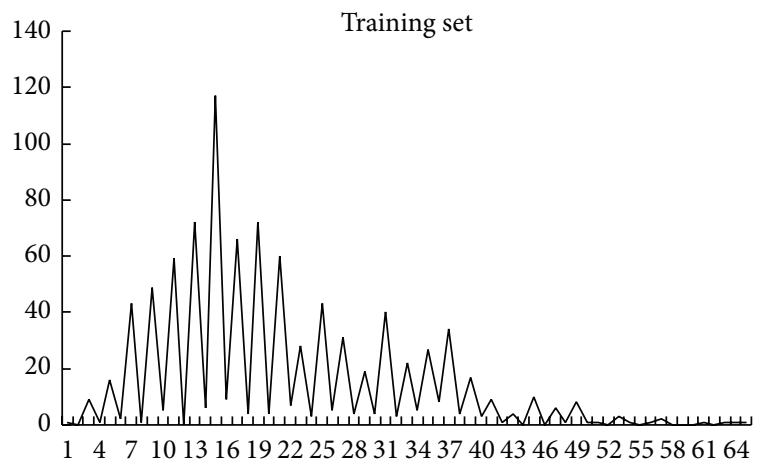

(a)

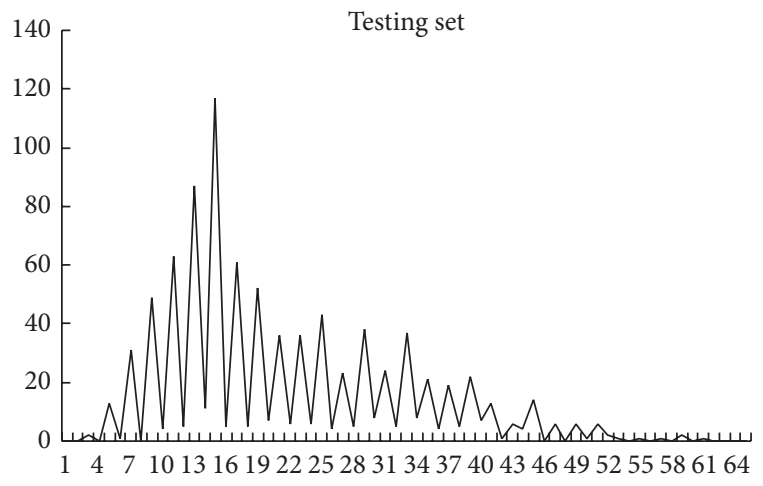

(b)

FIGURE 1: The numbers of sequences containing secondary structure segments. (a) and (b) are for training set and testing set, respectively.

CC variables describe the average interactions between different types of secondary structure segments, which can be calculated according to the following equation:

$$
\operatorname{CC}(i 1, i 2, \lg )=\sum_{j=1}^{L-\lg } \frac{\left(S_{i 1, j}-\bar{S}_{i 1}\right)\left(S_{i 2, j+\lg }-\bar{S}_{i 2}\right)}{(L-\lg )},
$$

where $i 1$ and $i 2$ are two different types of secondary structure segments (helix, strand, or coil) and $S_{i 1, j}$ is a property value of secondary structure segment $i 1$ at position $j . \bar{S}_{i 1}\left(\bar{S}_{i 2}\right)$ is the average property value for secondary structure segment $i 1(i 2)$ along the whole sequence. The dimension of CC variables is $3 * 2 * \lg$.

(2) The Selection of the Maximum Value of lg. The statistical analysis of the number of the secondary structure segments in the 27-class folds dataset is shown in Figure 1. The abscissa represents the number of secondary structure segments. The ordinate represents the number of sequences. The percentage of sequences that contained less than five secondary structure segments was below $0.5 \%$. The maximum value of $\mathrm{lg}$ was selected as $4(\max (\lg )=4)$.

(3) The Values of Physicochemical Properties. In this paper, four physicochemical properties used by many researchers were selected as ACC feature values to calculate the interactions of segments: hydrophobicity $(\mathrm{H} 1)$, hydrophilicity $(\mathrm{H} 2)$, polarity (PL), and solvent accessible surface area (SASA). The values of physicochemical properties for the 20 amino acid residues were taken from the literature of Guo et al. [36] (Table 2). ACC can reflect the segments-order and the longrange correlation information of the sequence, which has a major influence on protein folding.

2.2.4. Predicted Secondary Structure Information $(P)$. As the protein fold is a description based on the secondary structure, the formation of secondary structure in sequence influences the folding of protein. From the researches of published literatures $[17,21,22,25]$, in this paper, the occurrence frequencies of three kinds of predicted secondary structure segments motifs were extracted as a feature parameter; thus we can get a 3-dimensional vector. Then the occurrence frequencies of three-state of amino acid residues (i.e., helix, strand, and coil) were extracted as a feature parameter, and then we got another 3-dimensional vector. Therefore, the frequencies of secondary structure segments and three-state of amino acid residues above were converted into a 6-dimensional vector, which were represented by P. Here, the online web server PSIPRED (http://bioinf.cs.ucl.ac.uk/psipred/) was used to get the predicted secondary structure of each protein sequence.

2.3. Random Forest. Random Forest is an algorithm for classification developed by Breiman [38]. The basic idea of the algorithm is that multiple weak classifiers compose a strong individual classifier. Random Forest uses a collection of multiple decision trees, where each decision tree is a classifier, every split of the decision tree is a classifier, and the final predictions are made by the majority vote of trees. Random Forest has the following advantages: (1) few parameters to adjust and (2) data that does not require preprocessing. And Random Forest has two important parameters: (1) the number of feature parameters selected by each node of a single decision tree at each split, the number being represented by $m$ ( $m=\sqrt{M}$, where $M$ is the total number of features which were selected initially), and (2) the number of decision trees represented by $k$ (in this paper, $k=1000$ ). The Random Forest algorithm has been successfully used for the prediction of antifreeze proteins [39], DNA-binding residues [40], the metabolic syndrome status [41], $\beta$-hairpins [42], and so forth. Here, R-2.15.1 software (http://www.r-project.org/) was used to perform the Random Forest algorithm by calling the randomForest program package.

\section{Results and Discussion}

3.1. The Comparison Results with Different Parameters. For the 27-class fold dataset, amino acid composition, motif frequency, predicted secondary structure information, and the interaction of secondary structure segments were extracted as feature parameters, with the combined feature vector as input parameters for the Random Forest algorithm. The overall accuracy of the testing set in the dataset measured up to $78.38 \%$ by independent test.

For further comparison, identification results from the gradual addition of relevant feature parameters are listed (Table 3). Furthermore, the architecture of the protein folds identification system is shown (Figure 2). 
TABle 3: Prediction accuracies of different parameters in the testing set (\%).

\begin{tabular}{|c|c|c|c|c|c|c|c|}
\hline Fold & A & $\mathrm{A}+\mathrm{ACC}$ & $\mathrm{A}+\mathrm{ACC}+\mathrm{M}$ & $\mathrm{A}+\mathrm{ACC}+\mathrm{M}+\mathrm{P}$ & $\begin{array}{c}A+A C C+M+P \\
(5-\text { fold cross } \\
\text { validation })\end{array}$ & $\begin{array}{l}\text { The results of } \\
\text { Liu et al. [27] }\end{array}$ & $\begin{array}{c}\text { Ding and Dubchak's } \\
\text { dataset }[11] \\
A+A C C+M+P\end{array}$ \\
\hline 1 & 21.43 & 71.43 & 71.43 & 71.43 & $75.00(0.0252)$ & 78.5 & 100.00 \\
\hline 2 & 10.00 & 70.00 & 70.00 & 80.00 & $95.00(0.0000)$ & 90.0 & 100.00 \\
\hline 3 & 60.00 & 90.00 & 91.11 & 91.11 & $92.86(0.0026)$ & 75.5 & 75.00 \\
\hline 4 & 4.17 & 83.33 & 75.00 & 75.00 & $81.63(0.0000)$ & 54.1 & 87.50 \\
\hline 5 & 12.50 & 25.00 & 12.50 & 25.00 & $18.75(0.0187)$ & 25.0 & 77.78 \\
\hline 6 & 0.00 & 60.87 & 52.17 & 52.17 & $75.00(0.0342)$ & 39.1 & 66.67 \\
\hline 7 & 87.06 & 91.76 & 89.41 & 90.59 & $89.47(0.0114)$ & 82.3 & 79.55 \\
\hline 8 & 11.11 & 27.78 & 27.78 & 38.89 & $41.67(0.0000)$ & 55.5 & 75.00 \\
\hline 9 & 45.83 & 50.00 & 50.00 & 58.33 & $70.83(0.0421)$ & 70.8 & 84.62 \\
\hline 10 & 23.53 & 35.29 & 47.06 & 52.94 & $57.14(0.0255)$ & 47.0 & 66.67 \\
\hline 11 & 24.39 & 56.10 & 48.78 & 58.54 & $70.73(0.0185)$ & 43.9 & 37.50 \\
\hline 12 & 0.00 & 46.43 & 64.29 & 60.71 & $54.39(0.0096)$ & 60.7 & 89.47 \\
\hline 13 & 0.00 & 30.00 & 50.00 & 60.00 & $66.67(0.0426)$ & 10.0 & 50.00 \\
\hline 14 & 37.50 & 56.25 & 62.50 & 62.50 & $81.82(0.0000)$ & 75.0 & 25.00 \\
\hline 15 & 53.33 & 40.00 & 40.00 & 46.67 & $67.74(0.0136)$ & 40.0 & 100.00 \\
\hline 16 & 86.96 & 95.65 & 98.91 & 100.00 & $98.92(0.0144)$ & 89.1 & 66.67 \\
\hline 17 & 0.00 & 20.00 & 20.00 & 20.00 & $20.00(0.0097)$ & 20.0 & 91.67 \\
\hline 18 & 11.11 & 30.56 & 47.22 & 61.11 & $68.49(0.0894)$ & 16.6 & 38.46 \\
\hline 19 & 37.50 & 81.25 & 100.00 & 100.00 & $100.00(0.0300)$ & 81.2 & 62.96 \\
\hline 20 & 26.03 & 72.60 & 90.41 & 89.04 & $91.84(0.0398)$ & 87.6 & 41.67 \\
\hline 21 & 30.56 & 50.00 & 75.00 & 72.22 & $72.60(0.0217)$ & 52.7 & 75.00 \\
\hline 22 & 22.50 & 40.00 & 62.50 & 57.50 & $65.82(0.0113)$ & 50.0 & 41.67 \\
\hline 23 & 27.27 & 45.45 & 90.91 & 90.91 & $95.46(0.0107)$ & 78.7 & 57.15 \\
\hline 24 & 0.00 & 16.67 & 50.00 & 66.67 & $41.67(0.0373)$ & 50.0 & 25.00 \\
\hline 25 & 12.82 & 56.41 & 61.54 & 61.54 & $69.23(0.0233)$ & 30.7 & 12.50 \\
\hline 26 & 51.52 & 88.89 & 90.91 & 92.93 & $86.00(0.0104)$ & 67.6 & 62.96 \\
\hline 27 & 100.00 & 75.61 & 87.80 & 92.68 & $92.68(0.0122)$ & 1.000 & 96.30 \\
\hline Q & 43.66 & 68.80 & 76.25 & 78.38 & $81.16(0.0028)$ & 66.5 & 70.24 \\
\hline
\end{tabular}

Note: A means amino acid composition (20 dimensions), A + ACC means amino acid composition and the interaction of segments (164 dimensions), A + ACC + M means amino acid composition, the interaction of segments, and motif frequency ( 290 dimensions), and A + ACC + M + P means amino acid composition, the interaction of segments, motif frequency, and predicted secondary structure information (296 dimensions); $Q$ means the overall accuracy; the standard deviation values are in the parenthesis of the sixth column, the penultimate column is the results of Liu et al. [27] with the same dataset, and the last column is our results of the dataset built by Ding and Dubchak [11].

When only one feature parameter, amino acid composition, was used, the overall accuracy was $43.66 \%$ (Table 3 ). After adding the feature parameter based on the interaction of segments, the overall accuracy increased to $68.80 \%$ (a $25 \%$ higher overall accuracy). The accuracies for folds 1, 2, 4, 6, 19, and 20 increased more than $50 \%$, and the accuracies of folds $3,11,12,13,25$, and 26 increased approximately $30 \%$ except folds 15 and 27, and the accuracies of the remaining folds also improved in different degrees. The feature parameter based on the interaction of segments also had a great effect on the identification of protein folds. When adding the feature parameter of motif frequency to amino acid composition and interaction of segments, the overall accuracy increased to $76.25 \%$, an $8 \%$ higher overall accuracy. During this process, the accuracies of folds 12, 13, 19, 22, 23, and 24 increased considerably, and the accuracy of fold 27 showed improvement. Finally, adding the feature parameter based on predicted secondary structure information resulted in an 


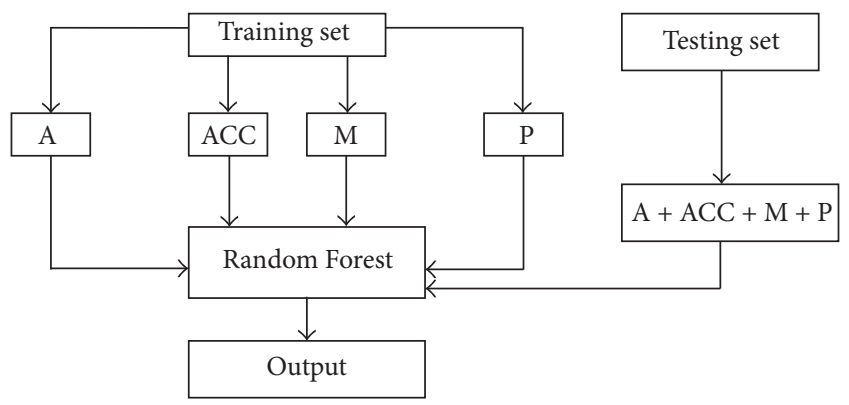

FIGURE 2: The architecture of the protein folds identification system.

overall accuracy of $78.38 \%$. Above all, as relevant feature parameters were gradually added, the accuracies of a large majority of folds improved in different degrees. Thus, the feature parameters used were very effective in predicting 27-class folds. According to the previous researches [16-18, 23], we combined the training set and testing set, and the corresponding prediction results and standard deviation (in parenthesis) by 5 -fold cross validation test were listed in the sixth column of Table 3 . With the same dataset, the previous results of Liu et al. [27] by independent test were also listed in Table 3 for comparison. We can see that the overall accuracy by independent test in our work was $12 \%$ higher than that of Liu et al. [27] (the penultimate column in Table 3), and the overall accuracy by 5 -fold cross validation test in our work was higher. The web server for protein folds prediction is accessible to the public (http://202.207.29.245:8080/Ha/HomePage/fzxHomePage.jsp).

3.2. The Comparison with Previous Results. To test the efficiency of our method, with the same feature parameters, classification strategy, and algorithm used above, the 27-class folds dataset built by Ding and Dubchak [11] was also tested. An overall accuracy of $70.24 \%$ was achieved by independent test (the last column in Table 3 ). The previous results from the same dataset are also listed in Table 4 for comparison. The accuracy was slightly lower than the results of Shen and Chou [24] and Yang et al. [25], but the overall accuracy in our work was higher than the accuracies of most previous researchers (Table 4).

3.3. The Identification Results of Structural Classifications. According to Shen and Chou's [21] description on structural classification in the literature, 27-class protein folds belong to four types of structural classification (Table 1). Therefore, the same feature parameters above were extracted and the combined feature parameters were inputted in Random Forest algorithm; the overall accuracy of the four types of structural classification in the testing set measured up to $92.55 \%$ by independent test. This overall accuracy was 3\% higher than that of Liu et al. [26] (Table 5). Our approach was also tested on the dataset built by Ding and Dubchak which has been used by many researchers and our results were superior to most previous results derived from the same dataset (Table 5).
TABLE 4: The previous identification results by an independent test from Ding and Dubchak's dataset (\%).

\begin{tabular}{llc}
\hline Author & Classifier & Accuracy \\
\hline Ding and Dubchak [11] & SVM (All-Versus-All) & 56.0 \\
Chinnasamy et al. [12] & Tree-Augmented Naive & 58.2 \\
Bayesian Classifier & \\
Shen and Chou [21] & OET-KNN & 62.1 \\
Nanni [13] & Fusion of classifiers & 61.1 \\
Chen and Kurgan [22] & PFRES & 68.4 \\
Guo and Gao [14] & GAOEC & 64.7 \\
Damoulas and Girolami [15] & Multiclass multikernel & 70.0 \\
Zhang et al. [20] & Increment of diversity & 61.1 \\
Ghanty and Pal [23] & Fusion of different & 68.6 \\
Dong et al. [18] & AClassifiers & 70.1 \\
Shen and Chou [24] & PFP-FunDSeqE & 70.5 \\
Yang et al. [25] & MarFold & 71.7 \\
Liu et al. [27] & SVM & 69.8 \\
Our work & Random Forest & 70.2 \\
\hline
\end{tabular}

\section{Conclusion}

In early 2001, Ding and Dubchak built the 27-class folds dataset and started research on the identification of 27-class protein folds with multiple feature groups. Researchers have since been devoted to the improvement of feature parameters, algorithms, classification strategies, and the datasets for the identification of protein folds and have achieved good identification results. Based on previous researches, we combined sequence information, structural information, and functional information as input feature parameters of the Random Forest algorithm for protein folds identification and obtained better results. Therefore, the addition of segment interactions and motif information for recognizing 27-class protein folds is a valid and novel approach.

Given the same dataset, when different feature parameters are used, the same sequence can be correctly or falsely classified. Here, our approach achieved the improved results with the following possible reasons. First, in considering the correlation at the level of secondary structure segments, we calculate the interaction information of secondary structure segments, which reflects the segments-order and long-range correlation information of the sequence and has a major influence on protein folding. Second, in considering the local conservation of kernel structure in protein folds, motif information was extracted, including functional motifs and statistical motifs. Finally, the Random Forest algorithm is a combination classifier of convenience and high efficiency whose final classification results are decided by votes from decision trees.

In our future work, with the same parameters and classification strategy, testing methods (jackknife test, 10-fold cross validation, etc.) would be used. Different physiochemical 
TABLE 5: Overall accuracies of structural class using different approaches in the testing set (\%).

\begin{tabular}{llccccc}
\hline \multirow{2}{*}{ Dataset } & \multirow{2}{*}{ Author } & \multicolumn{2}{c}{ Structural class } & \multicolumn{2}{c}{ Accuracy } \\
& & $\alpha$ & $\beta$ & $\alpha / \beta$ & $\alpha+\beta$ & $\mathbf{8 2 . 6 8}$ \\
Liu and $\mathrm{Hu}[26]$ & Our work & $\mathbf{9 4 . 6 7}$ & $\mathbf{9 1 . 7 3}$ & $\mathbf{9 7 . 3 3}$ & $\mathbf{9 2 . 5 5}$ \\
& Liu and Hu [26] & 97.04 & 85.43 & 94.07 & 78.21 & 89.24 \\
\hline \multirow{2}{*}{ Ding and Dubchak [11] } & Our work & $\mathbf{8 3 . 6 0}$ & $\mathbf{8 8 . 8 9}$ & $\mathbf{8 2 . 5 2}$ & $\mathbf{6 6 . 1 3}$ & $\mathbf{8 1 . 9 8}$ \\
& Liu and Hu [26] & 86.89 & 88.03 & 83.22 & 59.68 & 81.46 \\
& Zhang et al. [20] & & & & \\
& Chinnasamy et al. [12] & & & & \\
\hline
\end{tabular}

properties also would be analyzed for the recognition of protein folds. Moreover, Precision-Recall curves or ROC curves for individual folds could be presented.

\section{Conflict of Interests}

The authors declare that there is no conflict of interests that would prejudice the impartiality of this scientific work.

\section{Acknowledgments}

This work was supported by the National Natural Science Foundation of China (30960090, 31260203), The "CHUN HUI" Plan of Ministry of Education, and Talent Development Foundation of Inner Mongolia.

\section{References}

[1] A. C. R. Martin, C. A. Orengo, E. G. Hutchinson et al., "Protein folds and functions," Structure, vol. 6, no. 7, pp. 875-884, 1998.

[2] C. A. Ross and M. A. Poirier, "Protein aggregation and neurodegenerative disease," Nature Medicine, vol. 10, pp. S10-S17, 2004.

[3] S. Sri Krishna and N. V. Grishin, "Structural drift: a possible path to protein fold change," Bioinformatics, vol. 21, no. 8, pp. 1308-1310, 2005.

[4] P. J. Thomas, B. Qu, and P. L. Pedersen, "Defective protein folding as a basis of human disease," Trends in Biochemical Sciences, vol. 20, no. 11, pp. 456-459, 1995.

[5] S. Lindquist, S. Krobitsch, L. Li et al., "Investigating protein conformation-based inheritance and disease in yeast," Philosophical Transactions of the Royal Society B: Biological Sciences, vol. 356, no. 1406, pp. 169-176, 2001.

[6] T. Scheibel, J. Bloom, and S. L. Lindquist, "The elongation of yeast prion fibers involves separable steps of association and conversion," Proceedings of the National Academy of Sciences of the United States of America, vol. 101, no. 8, pp. 2287-2292, 2004.

[7] J. Ma, R. Wollmann, and S. Lindquist, "Neurotoxicity and neurodegeneration when PrP accumulates in the cytosol," Science, vol. 298, no. 5599, pp. 1781-1785, 2002.

[8] J. Ma and S. Lindquist, "Conversion of PrP to a self-perpetuating PrPSc-like conformation in the cytosol," Science, vol. 298, no. 5599, pp. 1785-1788, 2002.

[9] S. Han, B. Lee, S. T. Yu, C. Jeong, S. Lee, and D. Kim, "Fold recognition by combining profile-profile alignment and support vector machine," Bioinformatics, vol. 21, no. 11, pp. 2667-2673, 2005.

[10] J. Söding, "Protein homology detection by HMM-HMM comparison," Bioinformatics, vol. 21, no. 7, pp. 951-960, 2005.
[11] C. H. Q. Ding and I. Dubchak, "Multi-class protein fold recognition using support vector machines and neural networks," Bioinformatics, vol. 17, no. 4, pp. 349-358, 2001.

[12] A. Chinnasamy, W. Sung, and A. Mittal, "Protein structure and fold prediction using tree-augmented naïve bayesian classifier," Journal of Bioinformatics and Computational Biology, vol. 3, no. 4, pp. 803-819, 2005.

[13] L. Nanni, "A novel ensemble of classifiers for protein fold recognition," Neurocomputing, vol. 69, no. 16-18, pp. 2434-2437, 2006.

[14] X. Guo and X. Gao, "A novel hierarchical ensemble classifier for protein fold recognition," Protein Engineering, Design and Selection, vol. 21, no. 11, pp. 659-664, 2008.

[15] T. Damoulas and M. A. Girolami, "Probabilistic multi-class multi-kernel learning: on protein fold recognition and remote homology detection," Bioinformatics, vol. 24, no. 10, pp. $1264-$ $1270,2008$.

[16] C. Lin, Y. Zou, J. Qin et al., "Hierarchical classification of protein folds using a novel ensemble classifier," PLoS ONE, vol. 8, no. 2, Article ID e56499, 2013.

[17] M. T. A. Shamim, M. Anwaruddin, and H. A. Nagarajaram, "Support Vector Machine-based classification of protein folds using the structural properties of amino acid residues and amino acid residue pairs," Bioinformatics, vol. 23, no. 24, pp. 3320-3327, 2007.

[18] Q. W. Dong, S. G. Zhou, and J. H. Guan, "A new taxonomybased protein fold recognition approach based on autocrosscovariance transformation," Bioinformatics, vol. 25, no. 20, pp. 2655-2662, 2009.

[19] J. F. Li, J. G. Wu, and K. Chen, "PFP-RFSM: protein fold prediction by using random forests and sequence motifs," Journal of Biomedical Science and Engineering, vol. 6, pp. 1161-1170, 2013.

[20] H. Zhang, X. Hu, and Q. Li, “The recognition of 27-class protein folds: approached by increment of diversity based on multicharacteristic parameters," Protein \& Peptide Letters, vol. 16, no. 9, pp. 1112-1119, 2009.

[21] H. B. Shen and K. C. Chou, "Ensemble classifier for protein fold pattern recognition," Bioinformatics, vol. 22, no. 14, pp. 17171722, 2006.

[22] K. Chen and L. Kurgan, "PFRES: protein fold classification by using evolutionary information and predicted secondary structure," Bioinformatics, vol. 23, no. 21, pp. 2843-2850, 2007.

[23] P. Ghanty and N. R. Pal, "Prediction of protein folds: extraction of new features, dimensionality reduction, and fusion of heterogeneous classifiers," IEEE Transactions on Nanobioscience, vol. 8, no. 1, pp. 100-110, 2009. 
[24] H. B. Shen and K. C. Chou, "Predicting protein fold pattern with functional domain and sequential evolution information," Journal of Theoretical Biology, vol. 256, no. 3, pp. 441-446, 2009.

[25] T. Yang, V. Kecman, L. Cao, C. Zhang, and J. Zhexue Huang, "Margin-based ensemble classifier for protein fold recognition," Expert Systems with Applications, vol. 38, no. 10, pp. 1234812355, 2011.

[26] L. Liu and X. Z. Hu, "Based on adding combined vectors of motif information and power spectral density for predicting 27-class protein folds," Acta Biophysica Sinica, vol. 6, pp. 225-233, 2010 (Chinese).

[27] L. Liu, X. Z. Hu, X. X. Liu, Y. Wang, and S. B. Li, "Predicting protein fold types by the general form of Chou's pseudo amino acid composition: approached from optimal feature extractions," Protein and Peptide Letters, vol. 19, no. 4, pp. 439-449, 2012.

[28] A. Ben-Hur and D. Brutlag, "Remote homology detection: a motif based approach," Bioinformatics, vol. 19, no. 1, pp. i26-i33, 2003.

[29] X. Wang, D. Schroeder, and D. Dobbs, "Automated datadriven discovery of motif-based protein function classifiers," Information Sciences, vol. 155, no. 1-2, pp. 1-18, 2003.

[30] Y. Wang, X. Z. Hu, L. X. Sun, Z. X. Feng, and H. Y. Song, "Predicting enzyme subclasses by using random forest with multicharacteristic parameters," Protein and Peptide Letters, vol. 21, pp. 275-284, 2014.

[31] E. de Castro, C. J. A. Sigrist, A. Gattiker et al., "ScanProsite: detection of PROSITE signature matches and ProRule-associated functional and structural residues in proteins," Nucleic Acids Research, vol. 34, pp. W362-W365, 2006.

[32] T. L. Bailey, B. Mikael, F. A. Buske et al., "MEME suite: tools for motif discovery and searching," Nucleic Acids Research, vol. 34, pp. 369-373, 2006.

[33] S. Wold, J. Jonsson, M. Sjöström, M. Sandberg, and S. Rännar, "DNA and peptide sequences and chemical processes mutlivariately modelled by principal component analysis and partial least-squares projections to latent structures," Analytica Chimica Acta, vol. 277, pp. 239-253, 1993.

[34] Y. Guo, M. Li, M. Lu, Z. Wen, and Z. Huang, "Predicting Gprotein coupled receptors-G-protein coupling specificity based on autocross-covariance transform," Proteins: Structure, Function and Genetics, vol. 65, no. 1, pp. 55-60, 2006.

[35] Z. Wen, M. Li, Y. Li, Y. Guo, and K. Wang, "Delaunay triangulation with partial least squares projection to latent structures: a model for G-protein coupled receptors classification and fast structure recognition," Amino Acids, vol. 32, no. 2, pp. 277-283, 2007.

[36] Y. Z. Guo, L. Z. Yu, Z. N. Wen, and M. L. Li, "Using support vector machine combined with auto covariance to predict protein-protein interactions from protein sequences," Nucleic Acids Research, vol. 36, no. 9, pp. 3025-3030, 2008.

[37] F. X. Jun, M. Wu, Z. H. You, X. M. Zhao, and X. L. Li, "Prediction of $\beta$-hairpins in proteins using physicochemical properties and structure information," Protein and Peptide Letters, vol. 17, no. 9, pp. 1123-1128, 2010.

[38] L. Breiman, "Random forests," Machine Learning, vol. 45, no. 1, pp. 5-32, 2001.

[39] K. K. Kandaswamy, K. Chou, T. Martinetz et al., "AFP-Pred: a random forest approach for predicting antifreeze proteins from sequence-derived properties," Journal of Theoretical Biology, vol. 270, no. 1, pp. 56-62, 2011.
[40] L. Wang, M. Q. Yang, and J. Y. Yang, "Prediction of DNA-binding residues from protein sequence information using random forests," BMC Genomics, vol. 10, article S1, supplement 1, 2009.

[41] F. S. de Edelenyi, L. Goumidi, S. Bertrais et al., "Prediction of the metabolic syndrome status based on dietary and genetic parameters, using Random Forest," Genes and Nutrition, vol. 3, no. 3-4, pp. 173-176, 2008.

[42] S. C. Jia and X. Z. Hu, "Using Random Forest algorithm to Predict $\beta$-hairpin motifs," Protein and Peptide Letters, vol. 18, no. 6, pp. 609-617, 2011. 

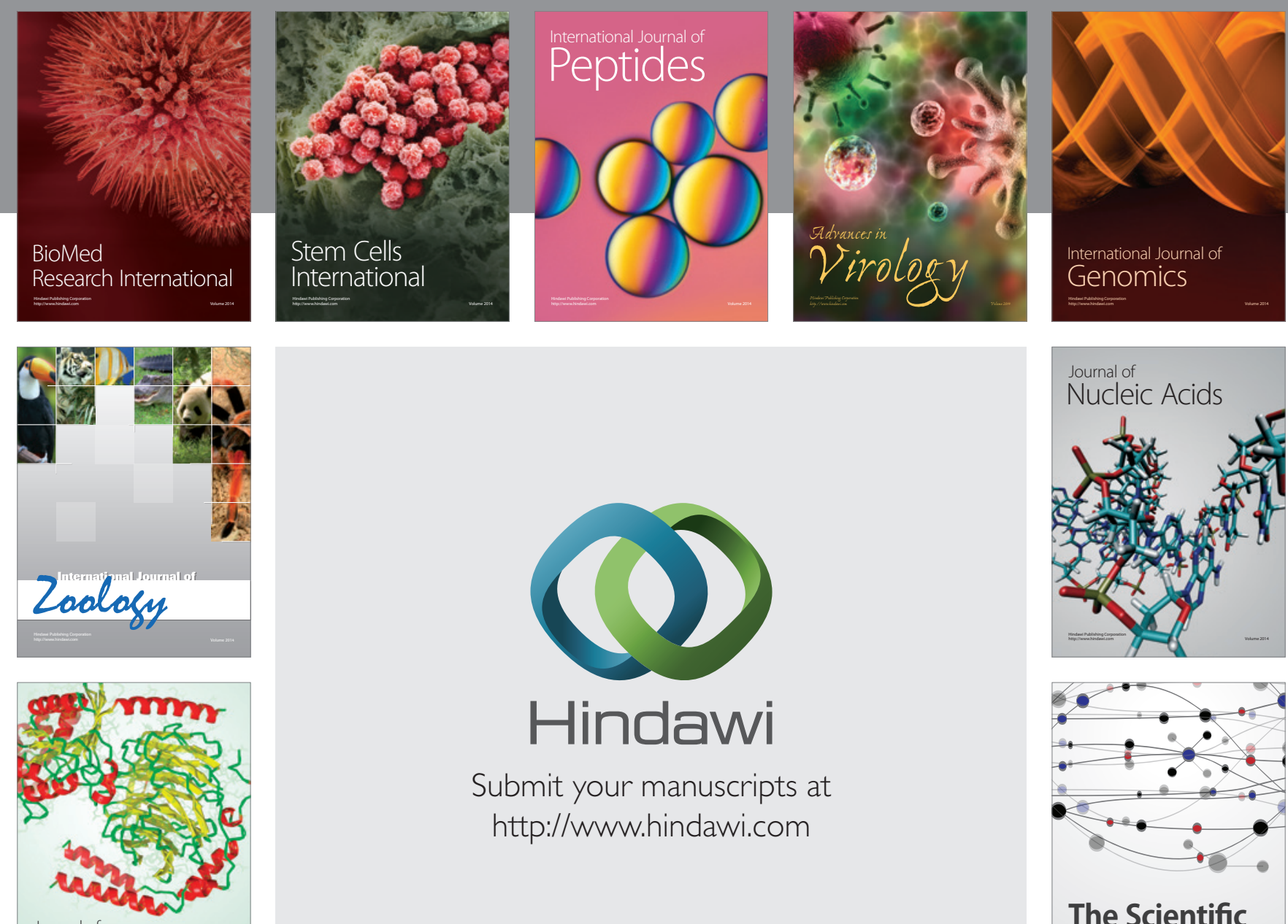

Submit your manuscripts at

http://www.hindawi.com

Journal of
Signal Transduction
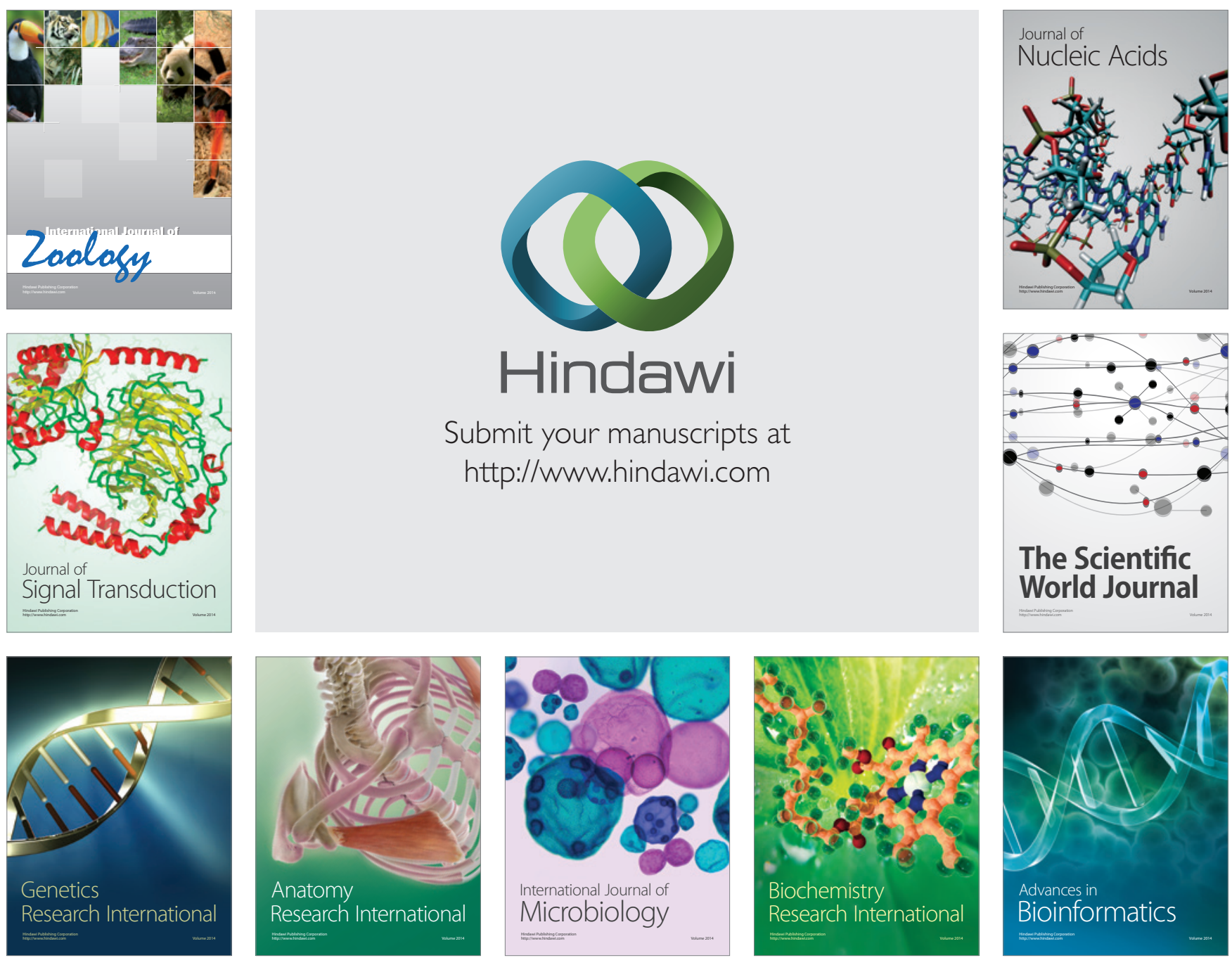

The Scientific World Journal
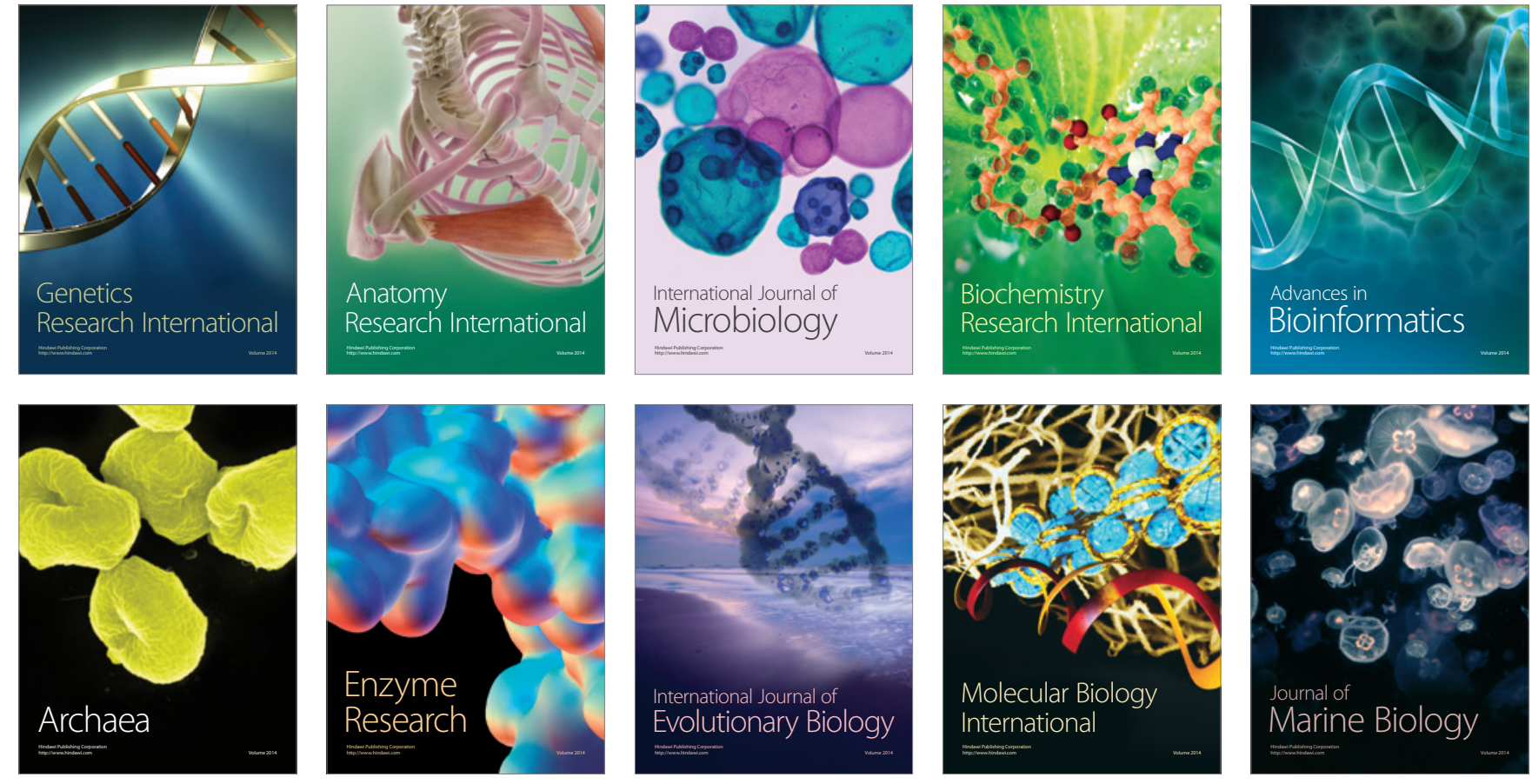